\title{
Scale and size effects in dynamic fracture of concretes and rocks
}

\author{
Y. Petrov ${ }^{1,2, a}$ and N. Selyutina ${ }^{1, b}$ \\ ${ }^{1}$ Saint Petersburg State University, 7/9, Universitetskaya nab., St. Petersburg 199034, Russia \\ 2 IPME RAS, Extreme States Dynamics Department, V.O., Bolshoj pr., 61, St. Petersburg 199178, Russia
}

\begin{abstract}
Structural-temporal approach based on the notion of incubation time is used for interpretation of strain-rate effects in the fracture process of concretes and rocks. It is established that temporal dependences of concretes and rocks are calculated by the incubation time criterion. Experimentally observed different relations between ultimate stresses of concrete and mortar in static and dynamic conditions are explained. It is obtained that compressive strength of mortar at a low strain rate is greater than that of concrete, but at a high strain rate the opposite is true. Influence of confinement pressure on the mechanism of dynamic strength for concretes and rocks is discussed. Both size effect and scale effect for concrete and rocks samples subjected to impact loading are analyzed. Statistical nature of a size effect contrasts to a scale effect that is related to the definition of a spatio-temporal representative volume determining the fracture event on the given scale level.
\end{abstract}

\section{Introduction}

The behaviour of the material strength dependent on the dimensions of the specimen is problem of fracture mechanics, construction, mining engineering and other fields engineering. To conduct of tests at the laboratory scale economically sound. The understandings of the translation data on material strength from microanalysis to macroexamination determine new concepts of material properties. These concepts allow to description of the material behaviour at any geometrical dimension.

At the equilibrium compression material is characterized by static strength; however, in the case of dynamic loading the failure stress is different depending on the strain rate. According to in [1-3], inertial confinement is the main response of increase of the fracture stress subject to dynamic loading relative quasi-static strength concrete. In this paper, it is proposed to use a notion of the incubation time, independent of inertial effect. As referred to in [4], the incubation time is a result of relaxation processes that precede the development of micro-structural defects in the material. It is a characteristic time of the fracture process invariably for any strain rate. On the basis of the incubation time criterion, it is possible to predict the behaviour of fracture stress over a wide range of rates.

The comparison of the fracture stress at quasistatic and dynamic conditions between two materials is conducted. It should be noted that material with low strength of material in case quasi-static tests involved higher strength on dynamic loading. Grote et al. [5] observed between specimens aggregated concrete and mortar. In [5], this phenomenon is explained by the acute effect on the plate impact constraints of compressive loads that inhibit crack growth, despite the quasi-static uniaxial compression increases the probability of cracking. It is interesting to note that in the static tests constraint loads also occur, but their influence on the impact plate

a e-mail: yp@YP1004.spb.edu

b e-mail: nina.selutina@gmail.com experiment is so great there, as described in [5]. In this paper is shown that the improvement of the concrete properties at high strain rates relative to mortar can be explained without the influence of the inertia effect (inertia at the micro-level of the material).

In [6] detailed the studying of the size effect is presented. Chasiotis and Knauss [6] observed the sensitivity of polysilicon films tensile strength to a change of specimen dimensions and identified by size and scale effects as the translation of data from micro-mechanical to macroscopic material properties and associated with the enormous influence of molecular or atomistic phenomena on the failure process of the material. It should be noted that notions of "size effect" and "scale effect" in [6] were nonselective. In this paper, "size effect" and "scale effect" concrete and rocks is considered based on structuraltemporal approach, described in [7-14]. The static compressive strength and the fracture stress decreases at the growth of specimen's length (size effect) and rises at the increases of specimen's width (scale effect). Petrov et al. [13] proposed, that the main characteristics of the material (incubation time and static strength) of incubation time criterion establish at a given scale level.

In the present research it is regarded results tests on Split Hopkinson bar of different rocks and concrete, obtained in [5,15-17]. According to structural-temporal approach, we evaluate the incubation time concrete and mortar plot dependences (high-speed curve) of the fracture stress on strain rate [5]. The different behaviour of concrete and mortar under static and dynamic loading is exhibited. Using fracture criterion we calculate incubation time and high-speed curve for each specimen of concrete [15] and rocks $[16,17]$. Comparisons of material strength of fixed length or width specimen are presented.

\section{Incubation time criterion}

The fracture criterion based on the concept of incubation time, proposed in [7-14], makes it possible to predict the 


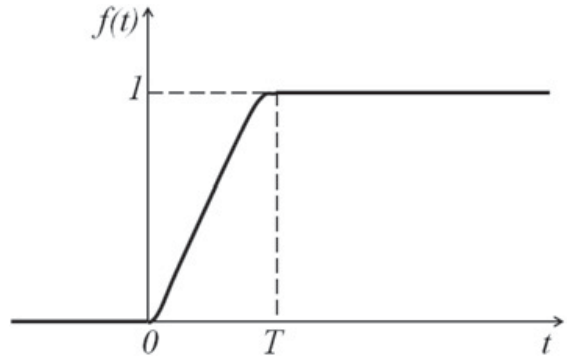

Figure 1. Schematic of fracture kinetics at the place of rupture.

unstable behaviour of the dynamic-strength characteristics observed in experiments on the dynamic fracture of solids. The fracture criterion can be written in the following general form:

$$
\frac{1}{\tau} \int_{t-\tau}^{t}\left(\frac{F\left(t^{\prime}\right)}{F_{c}}\right)^{\alpha} d t^{\prime} \leq 1 .
$$

Here, $F(t)$ is the intensity of the local force field causing the fracture (or structural transformation) of the medium, $F_{c}$ is the static limit of the local force field, and $\tau$ is the incubation time associated with the dynamics of the relaxation processes preceding the fracture. It actually characterizes the strain (stress) rate sensitivity of the material. The fracture time $t^{*}$ is defined as the time at which the equality sign is reached in Eq. (1). The parameter $\alpha$ characterizes the sensitivity of the material to the intensity (amplitude) of the force field causing the fracture (or structural transformation). Often, $\alpha=1$ gives a good agreement with test data.

One of the possible means of interpreting and determining the parameter $\tau$ is proposed here in the example of the mechanical rupture of a material. Let us assume that a standard test specimen made of the material in question is subjected to tension and is broken into two parts under a stress $P$ arising at a certain time $t=0: F(t)=P H(t)$, where $H(t)$ is the Heaviside step function. In the case of quasi-brittle fracture, the material would unload, and the local stress at the break point would decrease rapidly (but not instantaneously) from $P$ to 0 . In this case, a corresponding unloading wave is generated that propagates over the sample and can be detected by standard (e.g., interferometry) methods. The stress variation at the break point can be conditionally represented by the relation $\sigma(t)=P-P f(t)$, where $f(t)$ varies from 0 to 1 (Fig. 1 ) within a certain time interval T.

The case $f(t)=H(t)$ corresponds to the classical strength theory. In other words, according to the classical approach, rupture occurs instantaneously $(T=0)$. In practice, the rupture of a material (sample) is a process in time, and the function $f(t)$ describes the micro-scale level kinetics of the transition from a conditionally defectfree state $(f(0)=0)$ to a completely broken state at the given point $f\left(t^{*}\right)=1$ that can be associated with the macro-fracture event. On the other hand, application of the fracture criterion (1) to the macro-level situation $(F(t)=P H(t))$, gives the time to fracture $t^{*}=T=\tau$ at $P=F_{c}$. The physical meaning of incubation time is related to the relaxation process of growth of microdefects in the structure of material, which provides its nonreversible deformation. In this case characteristic time of relaxation can be considered as the incubation time [14]. This duration can be measured experimentally by statically fracturing the samples and controlling the rupture process by different possible methods, e.g., by measuring the time of the increase in pressure at the unloading wave front based on the recorded velocity profile of points (by interferometry) on the sample boundary.

\subsection{Definition fracture stress on incubation time criterion}

Let us present the application of the incubation time approach to a definition of strength under compression in a wide range of strain rate. As a rule, the concept of strength defines the growth limit value of the local stresses (maximum stress), at which the material does not fall into decay. In the incubation time approach, dynamic strength is characterized by the incubation time, but not maximum stress.

According to experimental data $[5,15]$ the criterion (1) rewritten for case the defect-free sample in the following form:

$$
\frac{1}{\tau} \int_{t-\tau}^{t}\left(\frac{\sigma\left(t^{\prime}\right)}{\sigma_{c}^{c o m p r}}\right)^{\alpha} d t^{\prime} \leq 1
$$

where $\sigma(s)$ is the time dependence of the average compressive stress in the specimen, $\sigma_{c}^{\text {compr }}$ is the static compressive strength of the material, and $\tau$ is the incubation time of material, $\alpha$ - parameter of sensitive to the level (or amplitude) of the intensity of the local stress and $\alpha \geq 1$.

According to the typical dependence of loading stress on time at the moment of fracture, described in $[5,15]$, an increase the compressive stress with strain rate can be assumed to be linear until it reaches the maximum value, so that

$$
\sigma(t)=E \cdot \dot{\varepsilon} \cdot t \cdot H(t)=\dot{\sigma} \cdot t \cdot H(t),
$$

where $H(t)$ is the Heaviside function, $E$ is Young's modulus, $\dot{\varepsilon}$ is the strain rate, $\dot{\sigma}$ is the stress rate.

The material fracture occurs if equality is attained in criterion (3). Defining an ultimate stress $\sigma_{d}$ as a stress at the moment of fracture $\sigma_{d}=\sigma\left(t_{*}\right)$, we obtain the function of limiting compressive stress on strain rate:

$$
\sigma_{d}(\dot{\varepsilon})=\left\{\begin{array}{l}
\left((\alpha+1)\left(\sigma_{c}^{\text {compr }}\right)^{\alpha} E \dot{\varepsilon} \tau\right)^{\frac{1}{\alpha+1}}, \dot{\varepsilon} \geq \frac{\sigma_{c}^{\text {compr }}}{E \tau}(\alpha+1)^{\frac{1}{\alpha}} \\
\sigma_{c}^{\text {compr }}+\left(1-\frac{1}{(\alpha+1)^{\frac{1}{\alpha}}}\right) E \dot{\varepsilon} \tau, \dot{\varepsilon}<\frac{\sigma_{c}^{\text {compr }}}{E \tau}(\alpha+1)^{\frac{1}{\alpha}} .
\end{array}\right.
$$

Grote et al. [5] were carried out plate impact experiments at strain rates of the order $10^{4}-10^{5} \mathrm{~s}^{-1}$ of concrete and mortar, and tests on a SHPB at strain rates of the order $10^{2}-10^{4} \mathrm{~s}^{-1}$ of mortar samples. It was determined during the quasi-static test the static strength of mortar specimens in compression $46 \mathrm{MPa}$ and Young's modulus $20 \mathrm{GPa}$. 


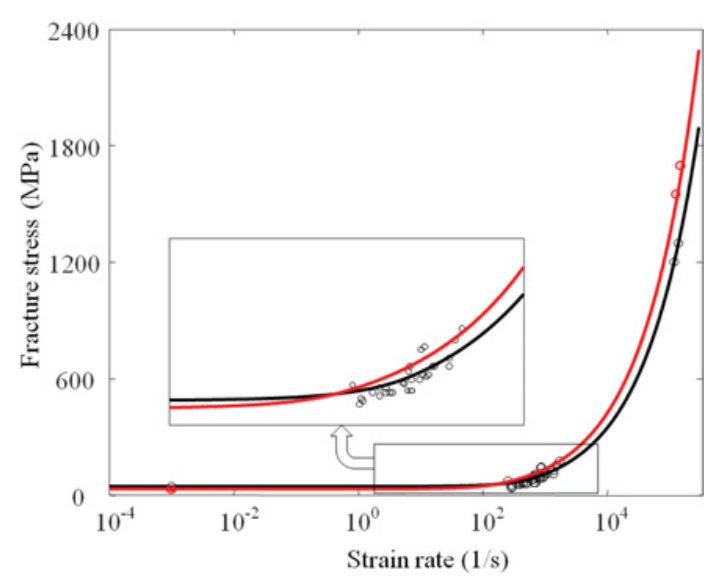

Figure 2. Compressive strength of concrete (red color) and mortar (black color) [5] as function of strain rate.

Table 1. Comparison of the behaviour of concrete and mortar in quasi-static tests and plate impact experiments [5].

\begin{tabular}{lllll}
\hline Material & $\sigma_{d}, \mathrm{MPa}$ & $E, \mathrm{GPa}$ & $\sigma_{c}^{\text {compr }}, \mathrm{MPa}$ & $\tau, \mu s$ \\
\hline concrete & 1700 & 45 & 30 & 6.5 \\
mortar & 1300 & 20 & 46 & 6.5 \\
\hline
\end{tabular}

Using the function (4) and the test data [5] is obtained the incubation time for the mortar and concrete specimens $\tau=6.5 \mu \mathrm{s}$ as an arithmetical middling from all samples. The dependence of the fracture stress on the strain rate (4) for concrete and mortar is plotted in Fig. 2. The comparison of mechanical properties two materials for test data [5] in quasi-static and dynamic experiments are presented in Table 1.

\section{Size and scale effect}

Let us consider the concept of the size effect is defined as a decrease of the material static strength in the specimen length growth and the concept of the scale effect is understood as an increase of the material static strength in the specimen width growth. Observe that notions of size and scale effects in [6] were nonselective and associated with the enormous influence of molecular or atomistic phenomena in the failure process of material and identified as the translation of data from micro-mechanical properties of material to macroscopic. In current paper is purposed that a change of the static strength dependent on material dimensions described of two different effects.

It is recalled that according to the "weakest link hypothesis", reported in [18], the static strength depends on the most damageable defect in the material under the application of a specific load. According to the statistical approach of strength (Weibull statistics), the massive body (or fiber) consists in set of structural elements and each component has own a local strength. In addition the fracture of a massive body (or fiber) is defined as elements group failure (or single element) under the application of a specific load.

The size effect can be explained using "weakest link hypothesis" [19]. The increase of sample length can be provided for the largest possibility of the propagation of the material defect. The growth of the defects is weakened

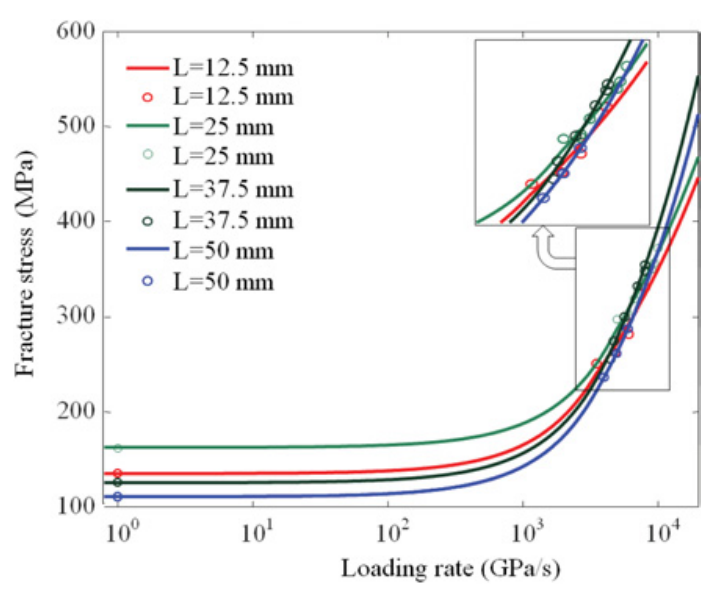

Figure 3. Compressive strength of Laurentian granite [16] as function of strain rate.

the strength properties of the material. It can be expected that response of the size effect result in growth of fracture probability in the same structure of the material.

Petrov et al. [20,21] and Morozov et al. [14] proposed the structural-temporal discretisation of the failure process and introduced the micro-structural fracture time (incubation time of the dynamic fracture process) and the spatial scale (characteristic size of the fracture zone) determined at a given scale level. According to the supposed concept in [13], fixed temporal and spatial characteristics of a scale level define lower and upper bounds of the characteristic size of the test specimen. Taking into account the change of material dimensions the failure characteristics of the incubation time approach can be modified within the limits only one or several failure levels. In the present paper the fracture level is characterized by incubation time calculated based on criterion (2).

Dai et al. [16] were conducted dynamic tests on compression for Laurentian granite cylindrical samples at loading rates 2000-10000 GPa/s. Samples of length $12.5 \mathrm{~mm}, 25 \mathrm{~mm}, 37.5 \mathrm{~mm}, 50 \mathrm{~mm}$ and diameter $25 \mathrm{~mm}$ were employed. Static strength $220 \mathrm{MPa}$ and Young's modulus $92 \mathrm{GPa}$ were determined in [16,22].

Static and SHPB tests on cylindrical specimens of granite, sandstone and limestone different dimensions $(D=22 \mathrm{~mm}$ and $L=11 \mathrm{~mm}, D=36 \mathrm{~mm}$ and $L=$ $18 \mathrm{~mm}, D=75 \mathrm{~mm}$ and $L=37.5 \mathrm{~mm}$ ) were undertaken in [17] at strain rate $36-360 \mathrm{~s}^{-1}$. Young's modulus of granite, sandstone and limestone is equal $63 \mathrm{GPa}, 5.4 \mathrm{GPa}$, $24 \mathrm{GPa}$, respectively.

On the basis of function (4), the dependence of the compressive strength on strain rate and estimated incubation time and loading parameter each specimen for rocks $[16,17]$ are presented in Fig. 3 - Fig. 6 and Table 2Table 3.

Figure 3 shows that the quasi-static strength and average fracture stress decrease in the growth specimen's length. The increase of the failure stress in the growth specimen's width is presented in Fig. 4 - Fig. 6.

Hao et al. [15] were performed quasi-static and SHPB compression tests in the range of strain rates $50-500 \mathrm{~s}^{-1}$ on cylindrical concrete samples with 
Table 2. Mechanical properties of Laurentian granite [16] dependent on specimen dimension.

\begin{tabular}{llll}
\hline $\mathrm{L}, \mathrm{mm}$ & $\mathrm{D}, \mathrm{mm}$ & $\tau, \mu \mathrm{s}$ & $\alpha$ \\
\hline 12.5 & 25 & 69.01 & 1.812 \\
25 & 25 & 58.96 & 1.836 \\
37.5 & 25 & 62.07 & 1.014 \\
50 & 25 & 64.3 & 1.07 \\
\hline
\end{tabular}

Table 3. Mechanical properties of rocks [16] dependent on specimen dimension.

\begin{tabular}{lclllllc}
\hline $\mathrm{L}$ & $\mathrm{D}$ & $\tau, \mu \mathrm{s}$ & $\alpha$ & \multicolumn{2}{l}{$\tau, \mu \mathrm{s}$} & $\alpha$ & \multicolumn{2}{l}{$\tau, \mu \mathrm{s}$} & $\alpha$ \\
$\mathrm{mm}$ & $\mathrm{mm}$ & granite & \multicolumn{4}{l}{ sandstone } & \multicolumn{2}{l}{ limestone } \\
\hline 11 & 22 & 23.2 & 1.04 & 393.7 & 1.08 & 146.8 & 1.06 \\
18 & 36 & 34.6 & 1.22 & 345.2 & 1.08 & 96.18 & 1.06 \\
37.5 & 75 & 94.9 & 1.26 & 1000 & 1.21 & 18.89 & 1 \\
\hline
\end{tabular}

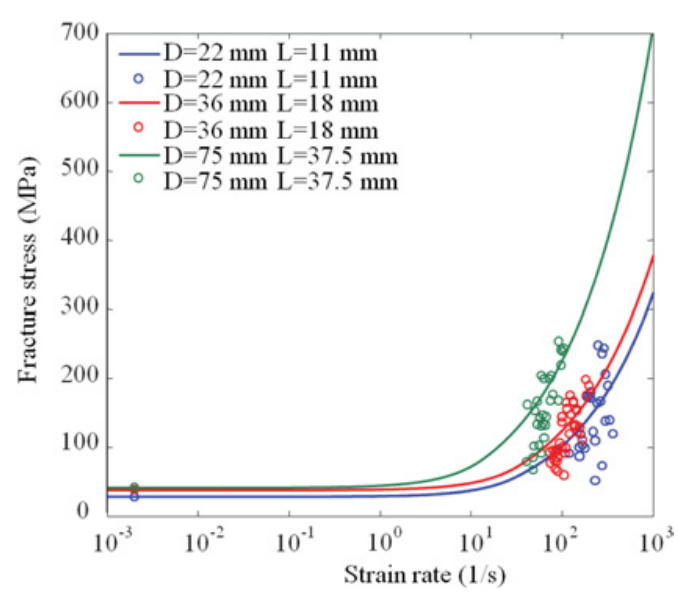

Figure 4. Compressive strength of granite [17] as function of strain rate.

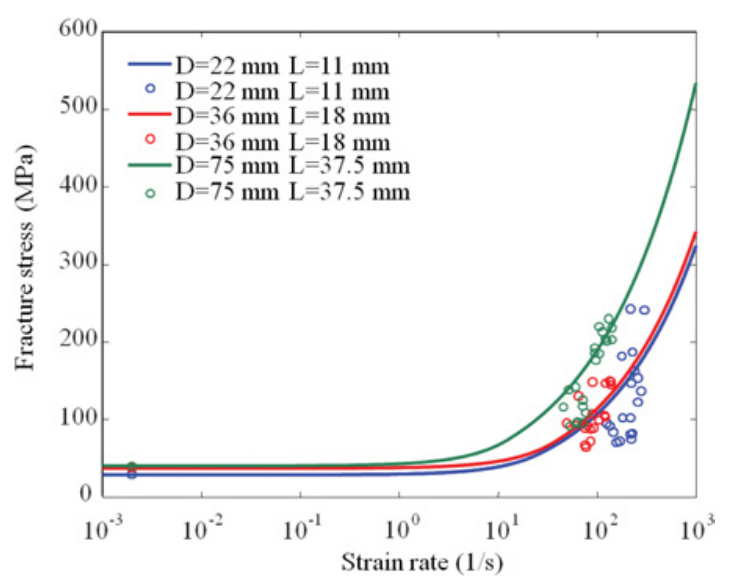

Figure 5. Compressive strength of sandstone [17] as function of strain rate.

different dimensions: $D=40 \mathrm{~mm}$ and $L=20 \mathrm{~mm}, D=$ $22 \mathrm{~mm}$ and $L=11 \mathrm{~mm}, D=32 \mathrm{~mm}$ and $L=20 \mathrm{~mm}$, $D=32 \mathrm{~mm}$ and $L=10 \mathrm{~mm}$. According to the bulk modulus and the shear modulus of concrete [15] is obtained Young's modulus $45 \mathrm{GPa}$. Table 4 shows static strength for each specimen, described in [15].

The results represented in the Table 4 show that an essential increase in sample diameter from $22 \mathrm{~mm}$

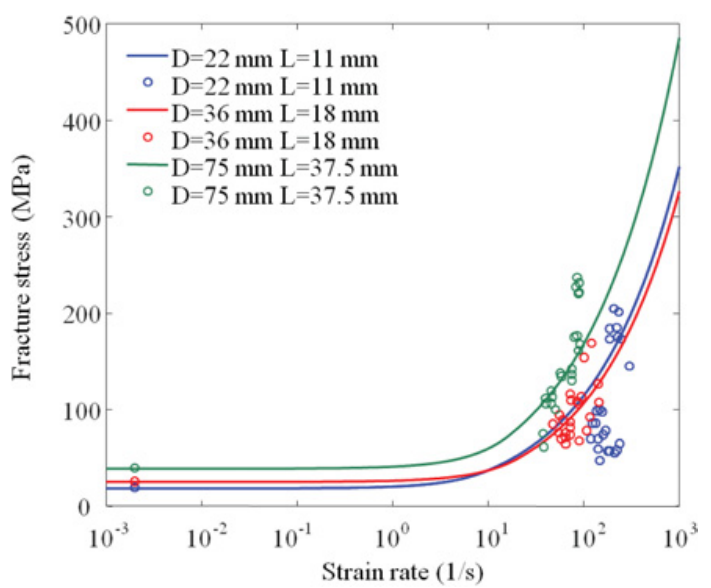

Figure 6. Compressive strength of limestone [17] as function of strain rate.

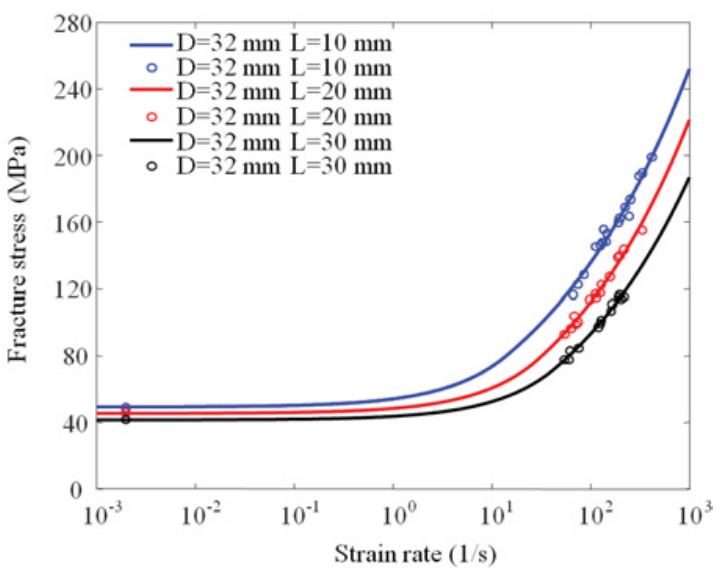

Figure 7. Experimental data [15] for concrete and dependences of compressive strength on strain rate at fixed width $32 \mathrm{~mm}$ and different lengths of the concrete specimen.

Table 4. Mechanical properties of concrete [15] dependent on specimen dimension.

\begin{tabular}{lccll}
\hline $\mathrm{L}, \mathrm{mm}$ & $\mathrm{D}, \mathrm{mm}$ & $\sigma_{c}^{\text {compr }}, \mathrm{MPa}$ & $\tau, \mu s$ & $\alpha$ \\
\hline 11 & 22 & 45.5 & 50.07 & 3.709 \\
10 & 32 & 48.8 & 128 & 2.703 \\
20 & 32 & 45.3 & 63.02 & 2.377 \\
30 & 32 & 41.2 & 40.54 & 2.301 \\
\hline
\end{tabular}

to $32 \mathrm{~mm}$ (at nearly the same length $10 \mathrm{~mm}-11 \mathrm{~mm}$ ) leads to a noticeable increase of measured static strength and incubation time. On the other hand the greater is the sample length for giving diameter, the less is the compressive strength as well as the material incubation time.

\section{Discussion}

Figure 2 - Fig. 6 show that average fracture stress is increased with strain rate. It should be noted that the critical comprehensive strength of material begins to increase drastically with a strain rate of the order $10^{2}-10^{3} \mathrm{~s}^{-1}$. According to high-speed curves, it can get an average value of the dynamic strength of the material at different loading rate or strain rates. This is phenomena 


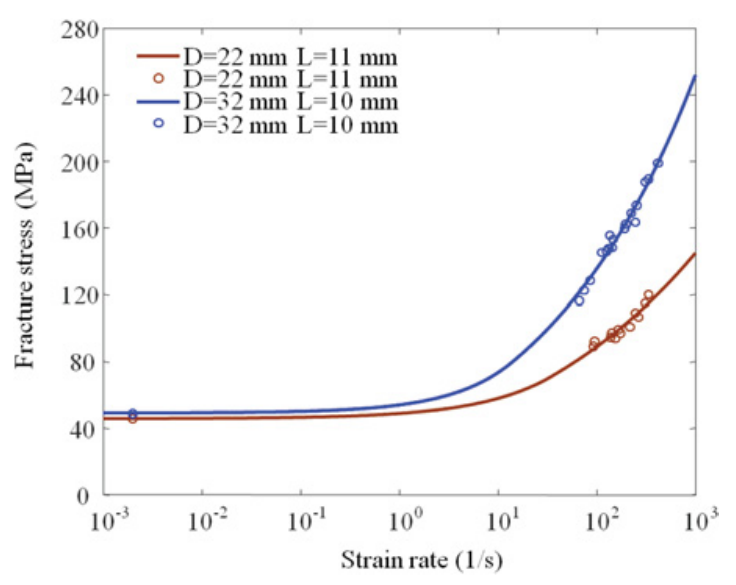

Figure 8. Experimental data [15] for concrete and dependences of compressive strength on strain rate at fixed length 10 (11) $\mathrm{mm}$ and different widths of the concrete specimen.

is fully explained by incubation time criterion at different values $\alpha$.

As can be seen from Table 1 the impact experiment [5] showed that concrete was stronger than mortar by $30 \%$.

Grote et al. [5] explain the effect of the existence of high confining pressure in the specimens. These stresses are growth drivers of micro-cracks in the static compression that critical compression stress reduces; also, these stresses are retard growth factors of microdefects in tests at high strain rates whereby material strength increases. Concrete is more inhomogeneous than in the case of high strain rates this influence of modulus becomes determinant mortar, so the fracture stress of mortar is less than that of concrete in plate impact experiments.

The results [5] in Table 1 demonstrate that the aggregated concrete has smaller static strength and larger elastic modulus compared to mortar. It is seen that the static compressive strength does not influence much of the critical stress at high strain rates of the order $10^{4}-$ $10^{5} \mathrm{~s}^{-1}$. Here it is considered that the local stress history follows the linear law (3) where Young's modulus defines the growth rate of compressive stress. Thus, it turns out that the competitive "strength inversion" between concrete and mortar is defined by the existence of incubation period and by the difference in elastic modulus. Taking in mind similar values of $\tau$ for mortar and aggregated concrete we get that the greater is Young's modulus the bigger is ultimate critical stress, which is attained during the incubation period. It has little effect at low impact velocities, but in the case of high strain rates this influence of modulus becomes determinant.

Figure 4 - Fig. 6 and Table 3 show that the behaviour of the incubation time in case increase in diameter and length of specimen is difficult. According to Table 2, incubation time of granite at a fixed diameter of the specimen is contained in the short range of 59-69 $\mu \mathrm{s}^{-1}$. The decrease of static strength in Fig. 3 can be associated with one scale level.

The different behaviour of static strength (known experimentally for each specimen concrete) at a fixed length and width of specimen is observed. Note that decrease and growth of the static strength of specimen are presented in Fig. 7 and Fig. 8, respectively. Table 4 shows that the growth of the incubation time and decrease of loading parameter are characterized by scale effect (in case of size effect, there will be a different (an opposite) relation between material strength and sample's sizes). Hence, variation of static strength can be exhibited using parameter material $\tau$. The set material parameters containing static strength and incubation time define spatial-temporal representative volume determining the fracture event on the given scale level.

\section{Conclusion}

The average critical compressive stress concrete and rocks at different loading rate and strain rate based on the structural-temporal approach was predicated using the incubation time of the fracture as a material parameter and coefficient of sensitive to amplitude of loading impulse.

The behaviour of both materials concrete and mortar over the course of quasi-static tests and plate impact experiments was investigated and compared. It is obtained that limiting compressive stress of mortar at a low strain rate was smaller than that of concrete, but at a high strain rate the opposite is true. It should be noted that the reason for this "strength inversion" effect can be the combined influence of incubation time and material elastic modulus along with the growth of strain rate.

Using experimental data from a split Hopkinson bar test was established incubation time for concrete, mortar samples of the different specimen dimension. On the basis of incubation time criterion characteristic curves in a wide range of strain rates were calculated. The static strength dependent on a sample length based on criterion (1) was evaluated. The size effect based on weakest link hypothesis was explained.

Size and scale effect as different phenomena was explained by structural-temporal approach. According to dynamic test data, definition of incubation time for different specimens can be predicated behaviour static strength of material. It is noted that concept of the size effect of static strength concerns a consideration of material failure in limit of one scale level. The physical meaning of the static strength of scale effect is defined as a transition between fracture levels.

This research was supported by the RFBR (14-01-00814) and Saint-Petersburg State University (grants 6.38.243.2014; 6.39.319.2014).

\section{References}

[1] H. Le Nard, P. Bailly. Mech Cohes-Frict Mater 5, 491-510 (2000)

[2] T. Tang, L.E. Malvern, D.A. Jenkins. ASCE J Eng Mech 118(1), 108-24 (1992)

[3] C. Huang, G. Subhash. J Mech Phys Solids 51, 1089-105 (2003)

[4] A.M. Bragov, Yu.V. Petrov, B.L. Karihaloo, A.Yu. Konstantinov, D.A. Lamzin, A.K. Lomunov, I.V. 
Smirnov. Engineering Fracture Mechanics 110, 477-88 (2013)

[5] D.L. Grote, S.W. Park, M. Zhou. Int J of Impact Eng 25, 869-86 (2001)

[6] I. Chasiotis, W.G. Knauss. J Mech Phys Solids 51, 1551-72 (2003)

[7] Yu.V. Petrov, A.A. Utkin. Mater Sci 25(2), 153-6 (1989)

[8] Yu.V. Petrov. Doklady Physics 49(4), 246-9 (2004)

[9] Yu.V. Petrov, E.V. Sitnikova. Technical Physics 49(1), 57-60 (2004)

[10] V. Bratov, Y. Petrov. International Journal of Fracture 146(1), 53-60 (2007)

[11] A.N. Berezkin, S.I. Krivosheev, Yu.V. Petrov, A.A. Utkin. Doklady Physics 45(11), 617-9 (2000)

[12] Yu.V. Petrov, P.A. Glebovski. Technical Physics 49(11), 1447-51 (2004)

[13] Y.V. Petrov, B.L. Karihaloo, V.V. Bratov, A.M. Bragov. International Journal of Engineering Science 61, 3-9 (2012)

[14] N. Morozov, Y. Petrov. Berlin-London-New York: Springer Verlag (2000)
[15] Y. Hao, H. Hao, G.P. Jiang, Y. Zhou. Cement and concrete research 52, 63-7 (2013)

[16] F. Dai, S. Huang, K. Xia, Z. Tan. Rock Mech Rock Eng 43, 657-66 (2010)

[17] L. Hong, X.B. Li, C.D. Ma, T. Yin, Z. Ye, G. Liao. Chin J Rock Mech Eng 27(3), 526-33 (2008)

[18] Yu.N. Rabotnov, Mechanics of solids (Moscow: Nauka, 1988) 712

[19] D.A. LaVan, T. Tsuchiya, G. Coles, W.G. Knauss, I. Chasiotis, D. Read. Cross Comparison of Direct Strength Testing Techniques on Polysilicon Films. In: Muhlstein, C., Brown, S.B. (Eds.), Mechanical Properties of Structural Films, ASTM STP 1413. American Society for Testing and Materials, West Conshohocken, PA, 1-12 (2001)

[20] Y. Petrov, N. Morozov. ASME Journal of Applied Mechanics 61, 710-2 (1994)

[21] Y.V. Petrov. Doklady Akademii Nauk USSR 321(1), 66-8 (1991)

[22] M.H.B. Nasseri , B. Mohanty, P.-Y.F. Robin. Rock Mech Min Sci 42, 450-60 (2005) 